\title{
Temperature Profiles in a Typical River-Aquifer Interaction Scenario
}

\author{
José Antonio Jiménez-Valera ${ }^{1}$, Francisco Alhama ${ }^{2}$ \\ ${ }^{1}$ Department of Mining and Civil Engineering / Technical University of Cartagena \\ Paseo Alfonso XIII, n52 - 30203, Cartagena, Spain \\ ${ }^{2}$ Applied Physics Department / Technical University of Cartagena \\ jose.jvalera@upct.es; paco.alhama@upct.es
}

\begin{abstract}
The river-aquifer interaction in a 2-D scenario, in which heat transfer is coupled with water flow coming from the river to the aquifer, is numerically solved by the network simulation method providing the temperature-depth profiles. As regards temperature, seasonal (harmonic) variations are assumed at the soil surface, a free condition is imposed at the right boundary of outflow and a constant temperature is set at the bottom of the domain. For the flow, a permeable condition (reflecting a large domain) is imposed at the limits of the aquifer. The complexity of the temperature and stream function patterns, as well as the change in the standard (no flow) vertical and horizontal profiles is shown. From these patterns, some guides for the setting of the inverse problem that search the water flow from the river to the aquifer are proposed.
\end{abstract}

Keywords: Hydrogeology, river-water interactions, aquifer recharge, inverse problem, numerical model, seasonal temperature profiles.

\section{Introduction}

Interaction between surface and groundwater in 2-D scenarios is an issue of great interest in hydrogeology because of the importance of water resources stored in aquifers which are recharged at ground surface from rivers, lakes or surface rain runoff. The total quantity of water that receives the aquifer is, no doubt, the variable of main interest for the hydrologists. As the use of underground flow meters is expensive and requires many instrumentation, alternative methods based on temperature-profile measurements (in the form of inverse problem) have been proposed in the last years. These inverse problem protocols allow also the determination of other properties of the soil (Boyle and Saleem [1] and Taniguchi and Sharma [2]).

These transient problems are clearly separated within the literature in 1-D or 2-D scenarios. The first, referred to domains in which water recharge (or discharged) is vertical, present analytical solution that are relatively easy to implement (Bredehoeft and Papadopulos [3]). In contrast, the 2-D domains, for which the flow coming from river o lakes has vertical and horizontal components, present a complex coupling between heat transfer and fluid flow without analytical solution. Most of papers that deal this problem do not report general solutions that allow searching a clear protocol for the inverse problem; instead, they numerically solve site scenarios by the use of standard software (Kulongoski and Izbicki [4]).

In all 1-D and 2-D cases, the papers assume seasonal (daily o annually) harmonic temperatures as the boundary condition at the soil surface and they take vertical, temperature profiles as the input data for the inverse problem. These profiles deviate from the standard profiles (derived from the absence of flow) according to the velocity flow field into the aquifer. The extension of the curved-region profile (either with or without water flow) is also dependent of the thermal properties of the soil and may extend to the whole depth in shallow aquifers. In addition, the flow boundary conditions at the bottom and vertical sides of the aquifer strongly influence the location at which temperature profiles can be read.

The complexity of the problem, which is far from its dimensional characterization, is evident since it's a coupled domain in which the number of physical (thermal and mechanical) and geometrical parameters involved is large.

This work, inspired in the paper of Duque, Calvache and Engesgaard [5], solved numerically the river-aquifer interaction in a large (symmetric) scenario, under seasonal temperature boundary condition at the surface and water-permeable boundaries at the limit of the aquifer. Numerical model, based on network method (Horno [6]), is easy to implement and provides reliable solutions with small computational times, as it has been demonstrated in other problems of similar or higher 
difficulty (Soto-Meca et al. [7]). Streamfunction and temperature profiles patterns within the aquifer allow an insight of the more suitable location for the temperature input data for setting the inverse problem.

\section{Physical, Mathematical and Network Models}

The physical, symmetric scheme of the problem is depicted in Figure 1. Temperature and flow boundary conditions are also shown in this figure. A river with constant hydraulic (height) head discharges on the sub-soil large aquifer whose bottom and vertical (right) boundary are permeable (outflow water) while the left boundary is impermeable (no flow).

As regards temperature, a seasonal (annual) harmonic temperature is imposed at the upper region exposed to environment while the temperature of the river-water is assumed constant. Vertical left boundary is adiabatic to reflect the symmetry of the scenario, the bottom maintains a constant value temperature and finally, the right vertical boundary is free to flow (heat is dragged by the water according to its temperature at the boundary).

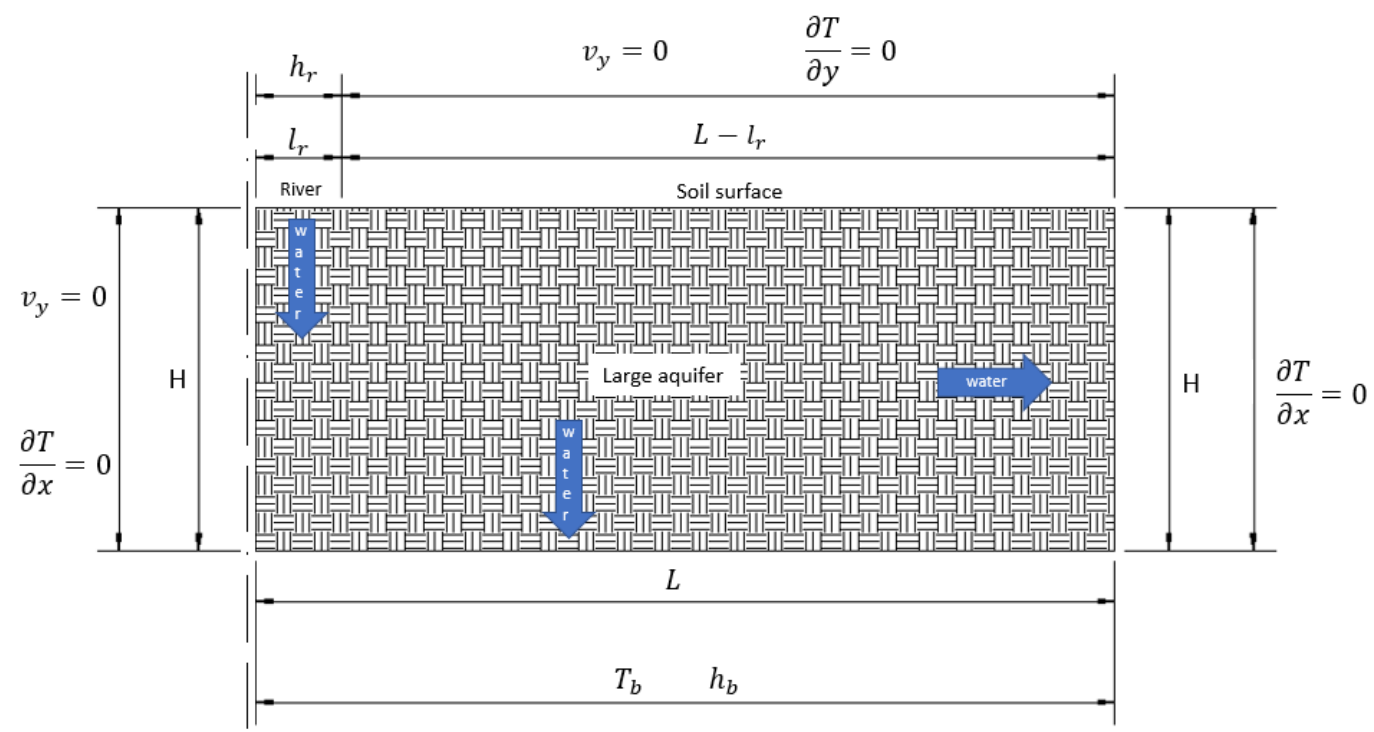

Figure 1. Physical scheme of the problem plus mechanical and heat boundary conditions

As for the mathematical model, rectangular coordinates with the origin at the upper-left corner of the aquifer are chosen (see Figure 1). The list of equations is as follows

$$
\begin{aligned}
& v_{x}=k_{x} \frac{\partial h}{\partial x} \\
& v_{y}=k_{y} \frac{\partial h}{\partial y}
\end{aligned}
$$

Eq. (1), Darcy constitutive law, is decoupled from the heat transfer so that it can be solved separately. In this equation, $\mathrm{k}_{\mathrm{x}}$ and $\mathrm{k}_{\mathrm{y}}(\mathrm{m} / \mathrm{s})$ are the horizontal and vertical hydraulic conductivities respectively, while $\mathrm{h}(\mathrm{J} / \mathrm{N})$ represents the piezometric head.

$$
c_{e} \rho_{e} \frac{\partial T}{\partial t}=k_{m}\left(\frac{\partial^{2} T}{\partial x^{2}}+\frac{\partial^{2} T}{\partial y^{2}}\right)-c_{w} \rho_{w}\left(v_{x} \frac{\partial T}{\partial x}+v_{y} \frac{\partial T}{\partial y}\right)
$$

Eq. (2), heat conservation flow, is the balance of the different heat flows (convection, conduction and storage) that locally balance one another. Fourier law is used for the conduction flow. The term $c_{e} \rho_{e}\left(\mathrm{calg}^{-10} \mathrm{C}^{-1}\right)$ is the specific heat of

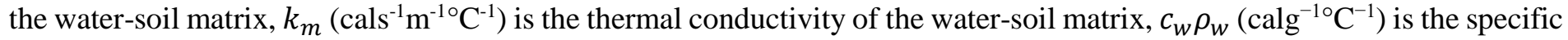


heat of water, $v_{x}$ and $v_{y}$ are the horizontal and vertical components of the water velocity, and $\mathrm{T}\left({ }^{\circ} \mathrm{C}\right)$ and $\mathrm{t}(\mathrm{s})$ the temperature and time variables.

$$
\begin{gathered}
h\left(0 \leq x \leq l_{r}, 0\right)=h_{r} \\
\left.\frac{\partial h}{\partial y}\right|_{\left(l_{r} \leq x \leq L, 0\right)}=0 \quad,\left.\quad \frac{\partial h}{\partial x}\right|_{(0, y)}=0 \\
h(x, H)=h(L, y)=h_{o}
\end{gathered}
$$

As regards the flow boundary conditions, Eq. (3a) is a first class (Dirichlet condition) condition for the head variable along the river. In this equation, $l_{r}(m)$ is the width of the river and $h_{r}(J / N)$ its piezometric head. Eq. (3b) is the no-flow condition at the ground surface and at the symmetric vertical wall, and Eq. (3c) is again a first class condition that emulates the constant head $h_{o}(J / N)$ at the outflow regions (bottom and right limits) of the aquifer. Temperature boundary conditions are described in Eqs. (4a), (4b) and (4c).

$$
\begin{gathered}
T(0 \leq x \leq h, 0)=T_{r} \\
T\left(l_{r} \leq x \leq L, 0\right)=T_{\text {med }} \sin (\omega t) \\
\left.\frac{\partial T}{\partial x}\right|_{(0, y)}=\left.\frac{\partial T}{\partial x}\right|_{(L, y)}=0 \\
T(x, H)=T_{o}
\end{gathered}
$$

A first class conditions at the river-water, Eq. (4a), where $T_{r}\left({ }^{\circ} \mathrm{C}\right)$ is the constant river temperature and at the ground surface. In Eq. (4b), surface soil temperature is dependent according to annual harmonic variation. A pure sinusoidal dependence has been chosen for simplifying. $\mathrm{T}_{\text {med }}\left({ }^{\circ} \mathrm{C}\right)$ is the average soil surface temperature while $\omega(\mathrm{rad} / \mathrm{s})$ is calculated as $\frac{2 \pi}{\tau}$, with $\tau$ the period of the oscillation. Homogeneous second class condition is imposed at the symmetric (adiabatic) line (no heat flow), Eq. (4c), while the right vertical boundary is assumed free, which means that flow dragged the heat according to the local temperature at the boundary. Temperature at the bottom $\mathrm{T}_{\mathrm{o}}$ is constant $\left({ }^{\circ} \mathrm{C}\right)$.

$$
T(t=0, x, y)=T_{\text {ini }}
$$

Finally, Eq. (5) represent the initial temperature, $\mathrm{T}_{\text {ini }}\left({ }^{\circ} \mathrm{C}\right)$.

This scenario has been adopted as typical without prejudice to the fact that real scenarios may be slightly or substantially different. Thus, it is common to find aquifers with an impermeable bottom and shallow aquifers, for which the boundary conditions are different. The same can be said of the initial temperature, which here is nothing more than a necessary variable (which does not affect the final solution of the thermal field). The problem described by the mathematical model represents a unique case in which the most important deviations of the temperature field occur in a region of the aquifer close to the river bank. Beyond this region, whose extension it would be interesting to determine, there are vertical temperature profiles where the recharge effect is not appreciated, as we will see later. These profiles represent the maximum or minimum value of the temperature that is reached at each point. From the mathematical point of view, the application of any other boundary condition does not present special difficulties from the point of view of numerical calculation.

The problem parameter values are listed in Table 1. 
Table 1: Parameter values of the problem

\begin{tabular}{|l|c|}
\hline $\mathrm{k}_{\mathrm{x}}(\mathrm{m} / \mathrm{s})$ & $6 \cdot 10^{-5}$ \\
\hline $\mathrm{k}_{\mathrm{y}}(\mathrm{m} / \mathrm{s})$ & $6 \cdot 10^{-5}$ \\
\hline$k_{\mathrm{m}}\left(\mathrm{cals}^{-1} \mathrm{~m}^{-1 \circ} \mathrm{C}^{-1}\right)$ & 1 \\
\hline $\mathrm{c}_{\mathrm{e}} \rho_{\mathrm{e}}\left(\mathrm{calg}^{-1 \circ} \mathrm{C}^{-1}\right)$ & $0.65 \cdot 10^{6}$ \\
\hline $\mathrm{c}_{\mathrm{w}} \rho_{\mathrm{w}}\left(\mathrm{calg}^{-1 \circ} \mathrm{C}^{-1}\right)$ & $1 \cdot 10^{6}$ \\
\hline $\mathrm{L}(\mathrm{m})$ & 50 \\
\hline $\mathrm{H}(\mathrm{m})$ & 20 \\
\hline $\mathrm{l}_{\mathrm{r}}(\mathrm{m})$ & 5 \\
\hline
\end{tabular}

\begin{tabular}{|l|c|}
\hline $\mathrm{h}_{\mathrm{r}}(\mathrm{J} / \mathrm{N})$ & 1 \\
\hline $\mathrm{h}_{\mathrm{o}}(\mathrm{J} / \mathrm{N})$ & 0 \\
\hline$\tau(\mathrm{s})$ & 31536000 \\
\hline$T_{r}\left({ }^{\circ} \mathrm{C}\right)$ & 0 \\
\hline$T_{\text {med }}\left({ }^{\circ} \mathrm{C}\right)$ & 1 \\
\hline $\mathrm{T}_{\mathrm{o}}\left({ }^{\circ} \mathrm{C}\right)$ & 1 \\
\hline$T_{\text {ini }}\left({ }^{\circ} \mathrm{C}\right)$ & 1 \\
\hline Amplitude $\left({ }^{\circ} \mathrm{C}\right)$ & 1 \\
\hline
\end{tabular}

The design of the numerical model follows the rules of the network simulation method, a tool largely employed in the literature to solve many types of coupled, non-lineal similar or even more difficult scenarios (such as Bénard, Yusa and many others) that the one here studied. Really, the numerical model is a simple modification of the software 'Fahet' (Alhama et al. [8]), developed by the research group of the UPCT and applied for the solution of some benchmark problems (Alhama et al. [9]). As we consider the network model as an auxiliary tool, we refer to the cited references to deepen in its design. In the crosslinking 100 X 20 cells have been used. The time step has been variable according to the smoothness of the solution. Other parameters of the simulation are pointed out later.

\section{Numerical Solution}

Iso-potential (hydraulic) and iso-temperature patterns are provided by the numerical model, while streamlines are determined with auxiliary computer routines. Figures 2 and 3, which preserve the geometric scale of the domain, show the patterns of iso-potentials and streamfunction, respectively.

It can be seen that, as expected, most of the flow is directed towards the bottom of the aquifer and in the area under the river. In fact, $90 \%$ of the total flow from the river is located in the region $0<\mathrm{x}<1_{\text {carac. }}$. However, it is important to note that neither the vertical nor the horizontal velocity of the fluid (which are relatively similar) in the left central region of the aquifer can be neglected, these give rise to strong couplings between the water and heat flows in this region. Beyond, to the right of the aquifer, the same phenomenon occurs, although with already negligible velocities, which induces standard vertical profiles practically independent of flow. On the other hand, it is noted that the discharge velocity at the bottom of the river changes with position, which confirms as the most suitable choice of the boundary condition that of constant hydraulic potential instead of that of constant velocity.

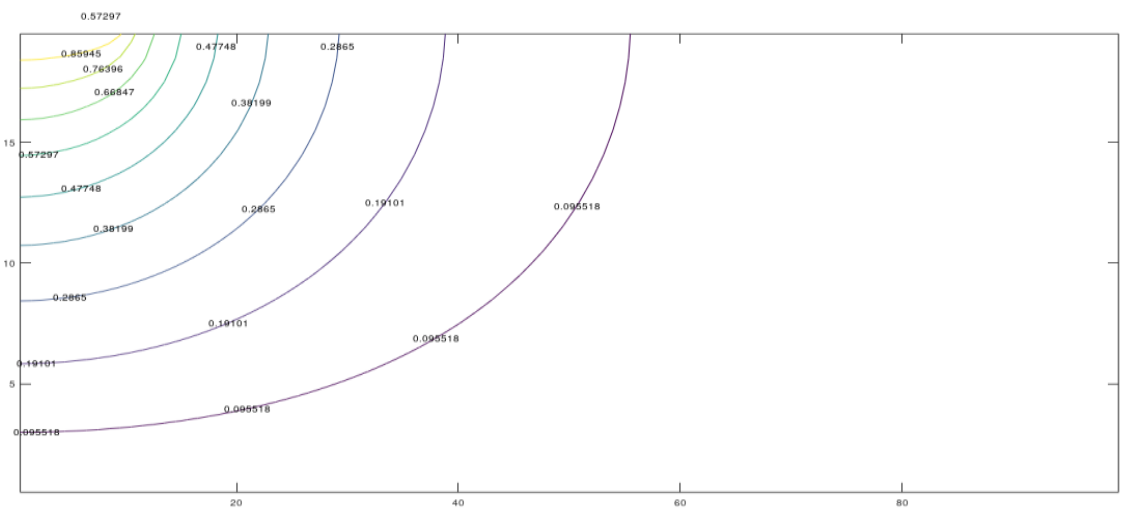

Figure 2. Lines of constant hydraulic potential. 


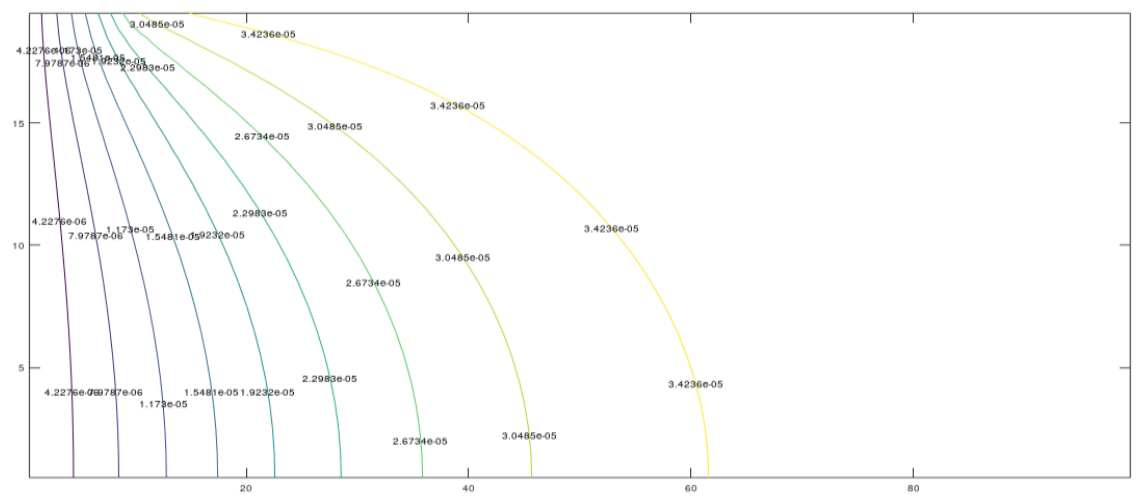

Figure 3. Stream lines pattern.

To illustrate the complex variation of the time-temperature patterns in which the harmonic displacement of the heat islands is represented, Figure 4 shows these patterns for nine different times within the same annual period, once the initial transient has been overcome.
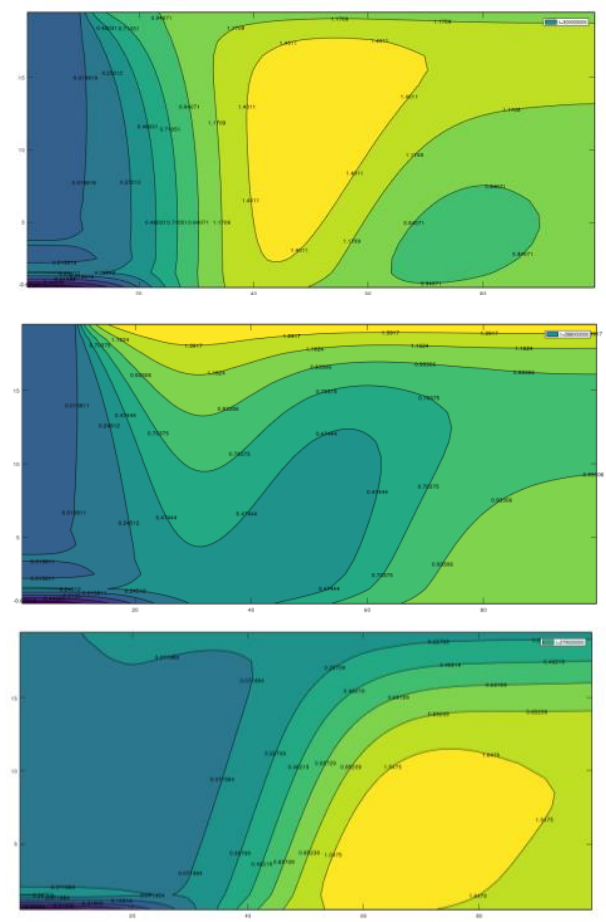
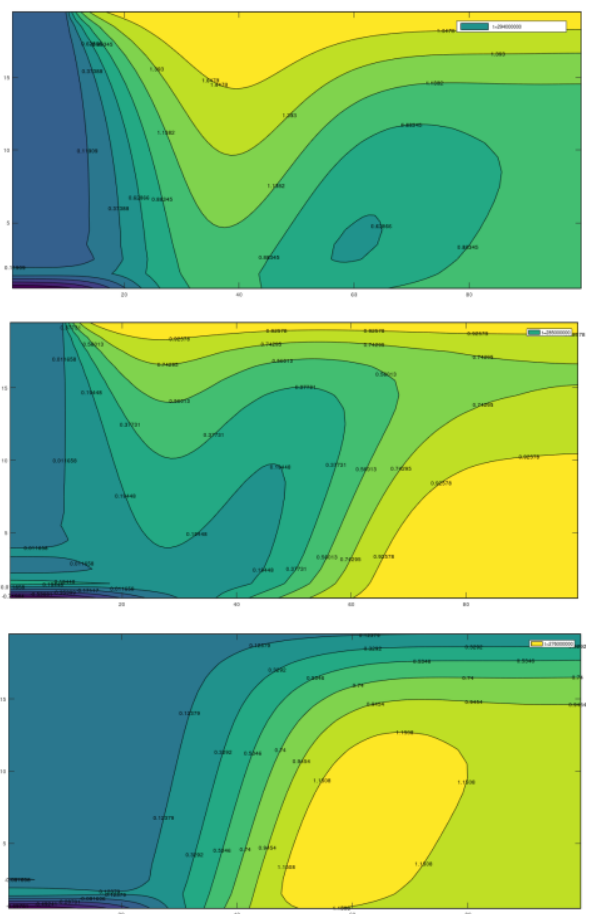
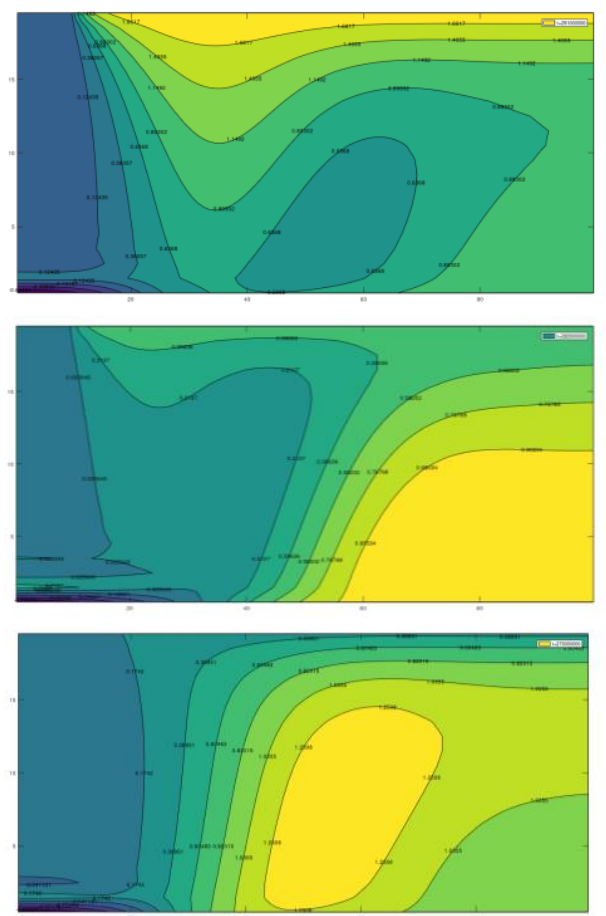

Figure 4. Isotemperature pattern corresponding to nine successive time intervals over a period.

These patterns are worth discussing from the point of view of their potential use as input data in inverse problems. In the first place, it is possible to imagine a regular evolution in the emergence, development and fading of the islands of hot and cold temperatures (close iso-line curves), which take place in the extensive central region of the aquifer. Despite the small velocities in the right domain, their effect on the temperature profile is important. A much larger extension of $L$ (for example, $200 \mathrm{~m}$ ) would have prevented the islands mentioned reach the right region.

Naturally, under the river, the temperature can be considered constant. Also, as a function of time within a season, the profiles of the aquifer region located immediately to the right of the river appear only for times when the soil temperature is 
close to its maximum or minimum values. Vice versa, in the times for which the environmental temperature is close to the maximum or minimum, the temperature profiles next to the river's edge appear well discriminated. Undoubtedly, all the above complexity would allow defining a pattern problem, with the most appropriate boundary conditions (like Yusa or Elder), for scenarios of this type.

\section{Temperature Profiles}

These profiles represent the maximum and minimum values of the thermal oscillation with depth. Figures 5 represents the maximum and minimum values of the temperature along different vertical columns within the aquifer, while Figure 6 shows their mean values. The relatively long extension of the aquifer is evident according to the shape of the profiles, whose tendency from column 90 (position $\mathrm{x}=44.75 \mathrm{~m}$ ) is to the standard profile type of zero water flow. It can be seen that in the first columns (up to positions of the order of cell 25) the maximum temperatures in the aquifer fall below $\mathrm{T}_{\text {med }}$ due to the effect of the cold water flow; the profiles in these regions are far from the standard and therefore could potentially serve to estimate the flow from them. The latter could also be said for the mean temperature profiles (Figure 6), all with a temperature lower than $\mathrm{T}_{\text {med. }}$. However, in very long aquifers, once a characteristic extension has been defined for which the temperature profiles move away from the standard zero flow profile, the previous figures could be considered as universal curves that occur in the same relative positions within this characteristic length, a very useful representation for the precise definition of inverse problems.

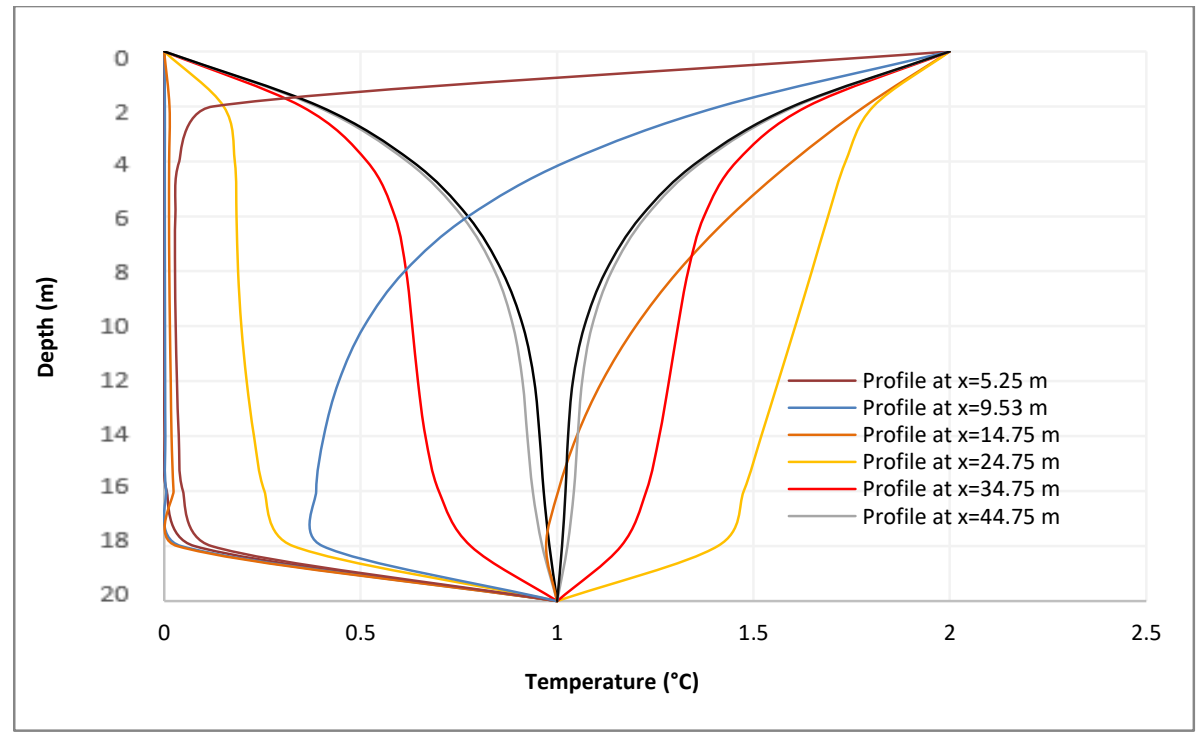

Figure 5. Maximum and minimum temperature depth-profiles 


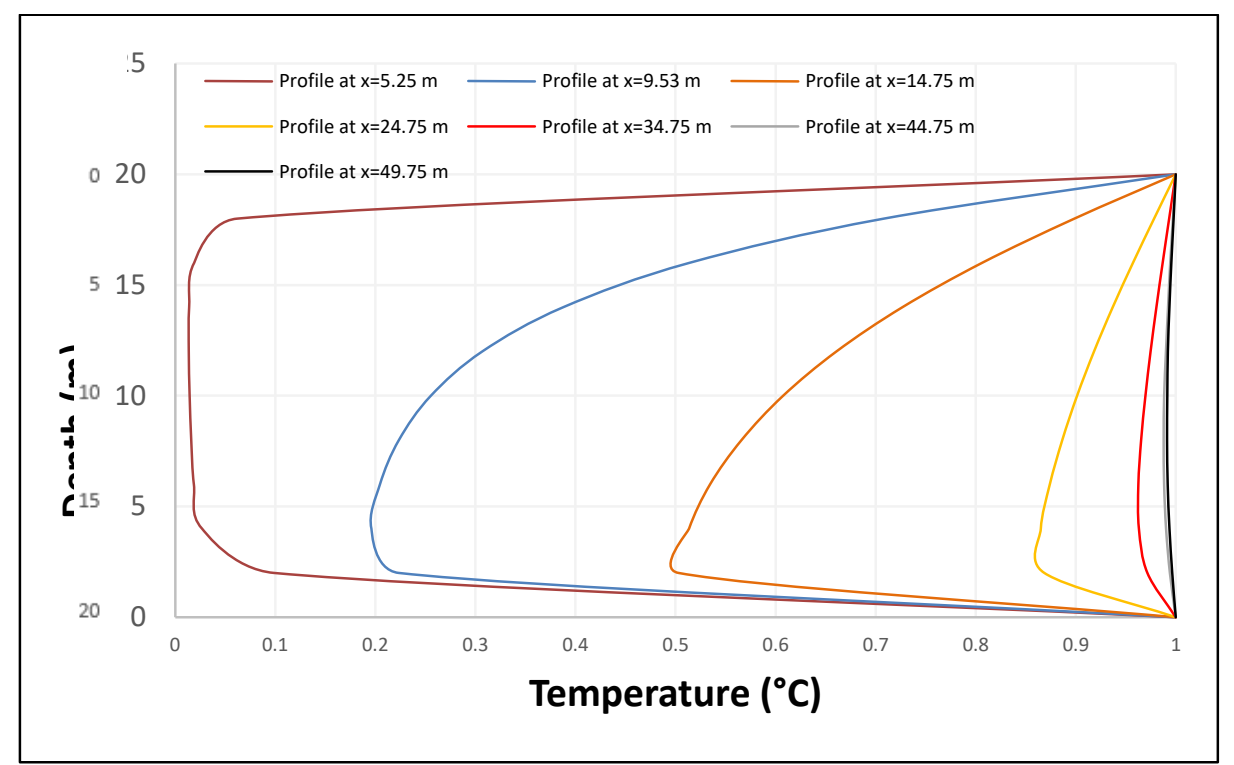

Figure 6. Average temperature depth-profiles

Figure 7 represents the profile of maximum deviations with depth. These deviations, very small next to the river, grow as we move away from it to a certain horizontal position in which they decrease and converge towards their standard values, all in coherence with the previous curves.

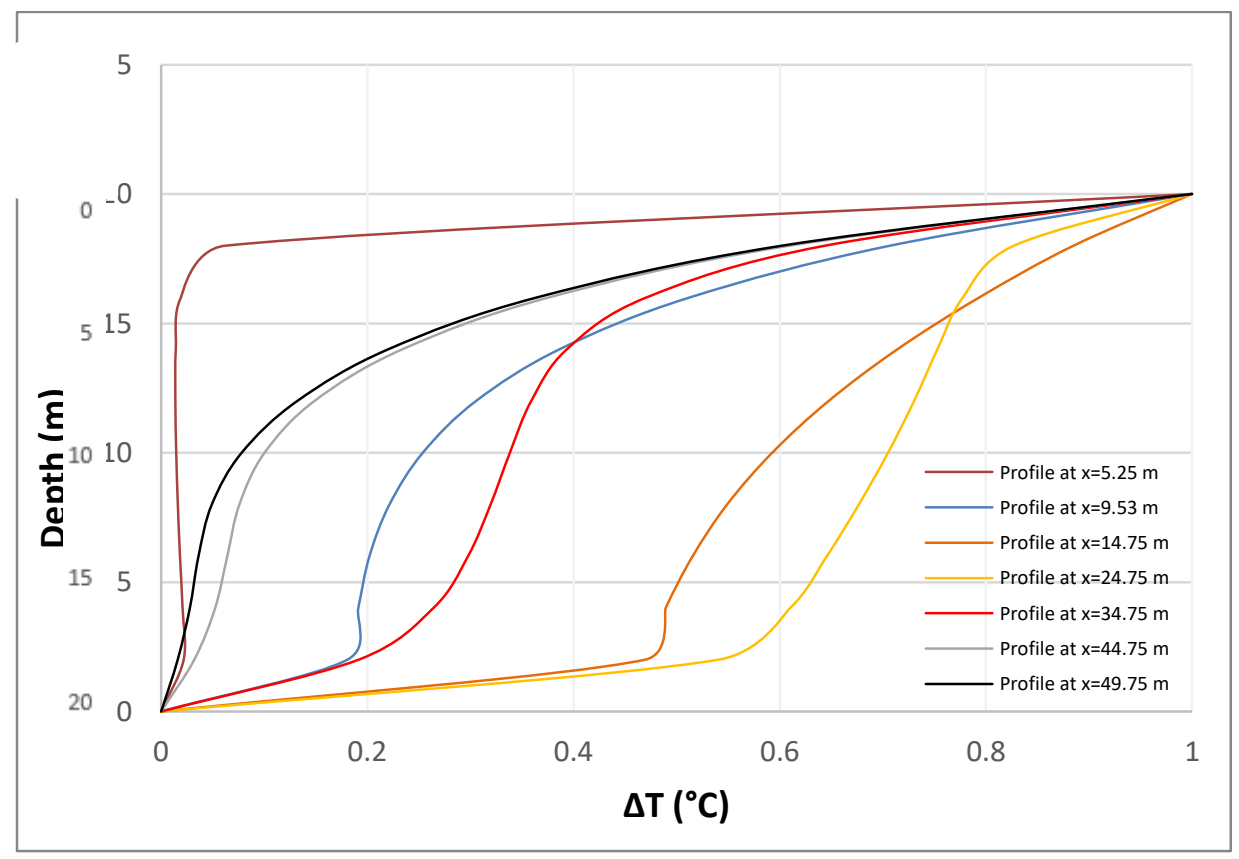

Figure 7. Maximum temperature deviations with depth

Finally, Figure 8 shows the horizontal temperature profiles in two typical positions, $y=5.5 \mathrm{~m}$ (row 15 (blue), near the surface) and $y=15.5 \mathrm{~m}$ (row 5 (green), near the bottom). Again these figures clearly denote the existence of a wide region 
for which these profiles move away from the stationary ones that would occur beyond the previously mentioned characteristic length. Its use for the inverse problem is relative, since it would require the installation of numerous wells.

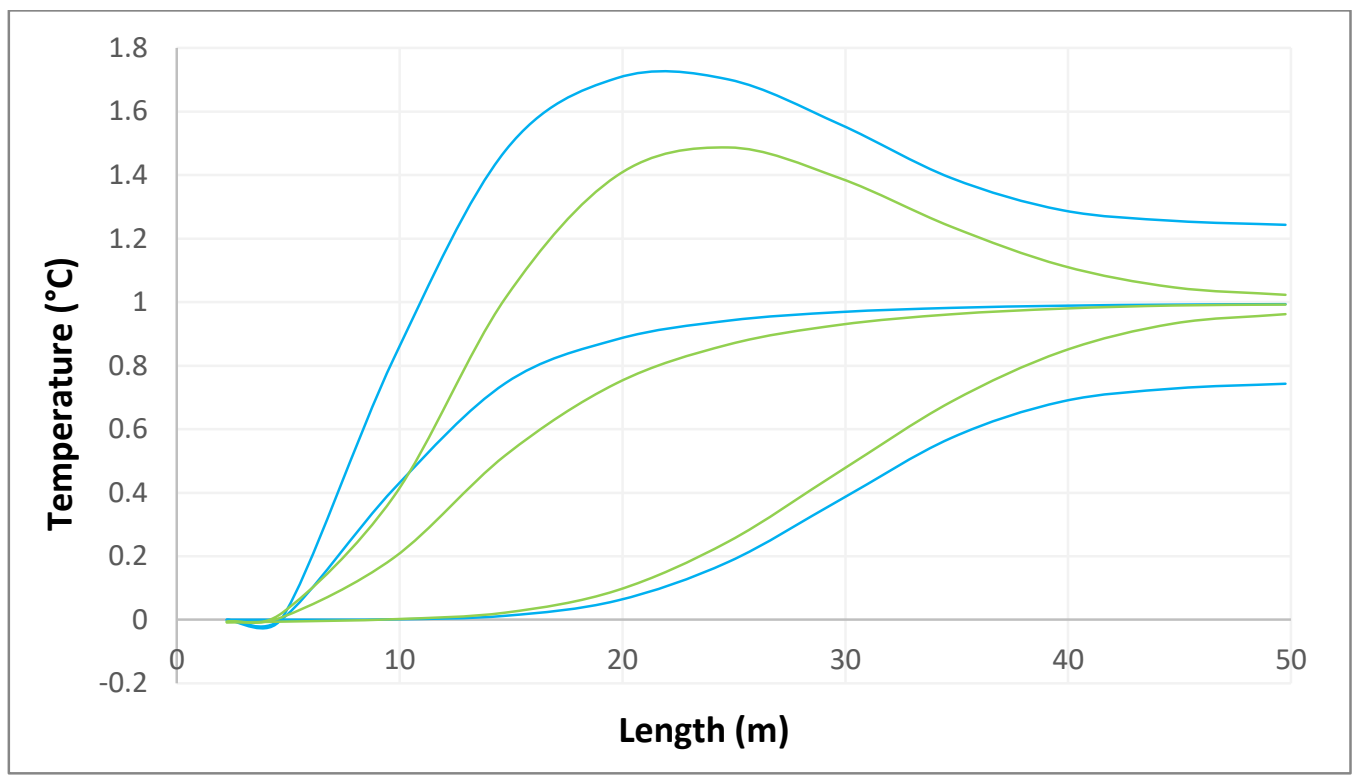

Figure 8. Horizontal temperature profiles.

\section{Final Comments and Conclusions}

The study of the recharge flows (and, eventually, discharge) of water to underground aquifers, coming from rivers or lakes, based on temperature measurements is a complex issue due to the multitude of variables involved and boundary conditions in these 2-D scenarios. Although numerically it is not a complex problem, its dimensional characterization and the presentation of universal results is still far from being stablished. The study carried out in this work is, in our view, an interesting step for the definition of standard scenarios that advance in the representation of universal solutions useful for proposing specific inverse problems in this field.

The proposed symmetric scenario presents an aquifer with a permeable bottom and vertical wall far from the river, as well as a seasonal harmonic temperature on the surface of the land in contact with the outside environment. Such conditions, especially the first, induce a region (or pattern) of flow essentially close to the edge of the river but with appreciable vertical and horizontal velocity components in the overall extension of the aquifer. This induces a very unique temperature pattern, with the existence of temperature islands (isotherms) that emerge, develop and fade over time in each annual season. Its development occupies practically the entire aquifer, which brings the horizontal characteristic length (significant of all changes in the pattern) closer to the length of the aquifer.

The temperature profiles (both horizontal and vertical) evolve uniformly to their 'pseudo' stationary values (associated with zero water velocities). After defining the vertical and horizontal characteristic lengths (for very long aquifers), it would be possible to set suitable criteria in order to determine the flow field from the temperature profiles data. It is a job that we hope to develop in the future.

\section{Acknowledgements}

We would like to thank "Fundación Séneca" for the scholarship awarded to José Antonio Jiménez Valera, which will allow us to continue this investigation. "21271/FPI/19. Fundación Séneca. Región de Murcia (Spain)" 


\section{References}

[1] J. M. Boyle and Z. A. Saleem, "Determination of recharge rates using temperature-depth profiles in wells," Water Resources Research, vol. 15, no. 6, pp. 1616-1622, 1979.

[2] M. Taniguchi and M. L. Sharma, "Determination of groundwater recharge using the change in soil temperature," Journal of Hydrology, vol. 148, no. 1-4, pp. 219-229, 1993.

[3] J. D. Bredehoeft and I. S. Papadopulos, "Rates of vertical groundwater movement estimated from the Earth's thermal profile," Water Resources Research, vol. 1, no. 2, pp. 325-328, 1965.

[4] J. T. Kulongoski and J. A. Izbicki, "Simulation of fluid, heat transport to estimate desert stream infiltration," Groundwater, vol. 46, no. 3, pp. 462-474, 2008.

[5] C. Duque, M. L. Calvache and P. Engesgaard, "Investigating river-aquifer relations using water temperature in an anthropized environment (Motril-Salobreña aquifer)," Journal of Hydrology, vol. 381, no. 1-2, pp. 121-133, 2010.

[6] J. Horno, "Network Simulation Method," Research Signpost, 2002.

[7] A. Soto-Meca, F. Alhama, and C.F. González-Fernández, "An efficient model for solving density driven groundwater flow problems based on the network simulation method", Journal of hidrology, vol. 339, pp. 39-53, 2007

[8] I. Alhama, A. Soto, M. Cánovas, F. Alhama, "FAHET: Flow and Heat Transport simulator", (C) 20042009 UPCT, 2011.

[9] I. Alhama, M. Cánovas, F. Alhama, "Simulation of fluid flow and heat transport coupled processes using FAHET software", Journal of porous media, vol. 18, no. 5, pp. 537-546, 2015. 